\title{
Electrically Detected Magnetic Resonance
}

O.M. FedOrYCH ${ }^{a}$, Z. WILAMOWSKI ${ }^{a}$, W. JANTSCH ${ }^{b}$

\author{
AND J. SADOWSKI ${ }^{c}$ \\ ${ }^{a}$ Institute of Physics, Polish Academy of Sciences \\ al. Lotników 32/46, 02-668 Warsaw, Poland \\ ${ }^{b}$ Institut für Halbleiterphysik, Johannes Kepler Universität, Linz, Austria \\ ${ }^{e}$ MAX-Lab, Lund University, Po. Box. 118, 22100 Lund, Sweden
}

\begin{abstract}
We compare the results of electrically detected magnetic resonance in a $2 \mathrm{D}$ electron gas in $\mathrm{Si} / \mathrm{SiGe}$ quantum wells with transport and magnetic resonance measurements on ferromagnetic $\mathrm{Ga}_{1-x} \mathrm{Mn}_{x} \mathrm{As}$. The results lead us to the conclusion that observation of electrically detected magnetic resonance is possible only in the case of a slow spin relaxation, where the microwave resonant absorption leads to a noticeable change of spin magnetization.
\end{abstract}

PACS numbers: 76.30.Pk, 72.25.Rb, 73.21.Fg

\section{Introduction}

Electrically detected magnetic resonance (EDMR) is one of the indirect methods to measure electron spin resonance (ESR) by detecting a change in some physical quantity induced by the magnetic resonance absorption. Very popular are indirect detection schemes, like optically detected magnetic resonance which monitors the change of photoluminescence caused by the magnetic resonance absorption. In the case of EDMR the change of electrical conductivity is monitored [1]. Two different methods are used. The electrical conductivity can be measured by a standard method with two current- and two voltage-contacts. Here both dc and/or ac voltages are used. Alternatively, also a contact-less method of the electrical measurement can be applied [2]. In that case the change of the microwave absorption caused by an induced electrical current adds to the direct absorption due to ESR. These two types of signals can be de-convoluted knowing the expected signal shapes and their dependence on experimental parameters, such as microwave power and temperature. In the standard low frequency method, the conductivity 
and its changes can be easily evaluated but the presence of connecting wires in the microwave cavity perturbs the microwave field. On the other hand, in the case of contact-less EDMR the absolute value of the conductivity is not known but the microwave field is not perturbed. Therefore, EDMR can be used to investigate resonance saturation and thus to evaluate the spin relaxation rate.

A detailed understanding of the EDMR mechanism is of some importance at present, because of possible applications in spintronic devices. More generally, EDMR offers experimental advantages:

(i) a simple electrical measurement allows one to observe the magnetic resonance,

(ii) the EDMR signal relates magnetic and electronic properties,

(iii) EDMR can be observed also in very small samples.

In this paper, we apply different EDMR methods for two types of samples. We compare the ESR of high mobility electrons in $2 d \mathrm{Si} / \mathrm{SiGe}$ quantum wells and the ferrimagnetic resonance in semiconducting, magnetic ( $\mathrm{Ga}, \mathrm{Mn}$ ) As layers. In fact, an EDMR signal in (Ga,Mn)As has not been found in contrast to our expectations, which were based on the fact that the dependence of the electric conductivity on the sample magnetization can be easily observed. These investigations allow us to formulate some general rules concerning the occurrence and amplitude of EDMR signals.

\section{Experimental results}

\subsection{Samples}

Electrons in Si/SiGe quantum wells [2] are characterized by Pauli paramagnetism and very weak spin-orbit coupling. The latter leads to low spin relaxation rates. As a consequence, the spin polarization of the electron gas is small due to degenerate carrier statistics, but it can be effectively changed under ESR conditions. The weak spin-orbit coupling should also result in a weak dependence of the electric conductivity on the spin polarization, but a clear dependence of conductivity on the layer magnetization is well evidenced by high field magnetoresistance experiments and is confirmed by our observation of a big EDMR amplitude. This dependence is caused by peculiar properties of a 2D metal [3].

In (GaMn)As alloys with a few percent of $\mathrm{Mn}$, magnetic order with a critical temperature exceeding $100 \mathrm{~K}$ occurs $[4,5]$. Magnetic resonance studies have shown that in the semi-metallic range the observed resonance corresponds to the low frequency mode of a ferrimagnetic resonance where the macroscopic magnetic moment is built from the coupled spins of $\mathrm{Mn}^{2+}\left(S_{\mathrm{Mn}}=5 / 2\right)$ ions and the spins of polarized holes [6]. The main contribution to the total magnetic moment comes from the Mn spins which are characterized by the weak spin-orbit coupling. The spin relaxation time of isolated $\mathrm{Mn}$ ions is very long. At low temperature it reaches 
milliseconds. However, the $p-d$ coupling of Mn $d$-spins to the $p$-hole spins, which causes spin splitting of the hole band and leads to magnetic ordering, also opens an additional channel for fast spin relaxation of the Mn $d$-states.

A comparison of the low frequency magneto-resistance, microwave absorption, and the EDMR signal are shown in Fig. 1.

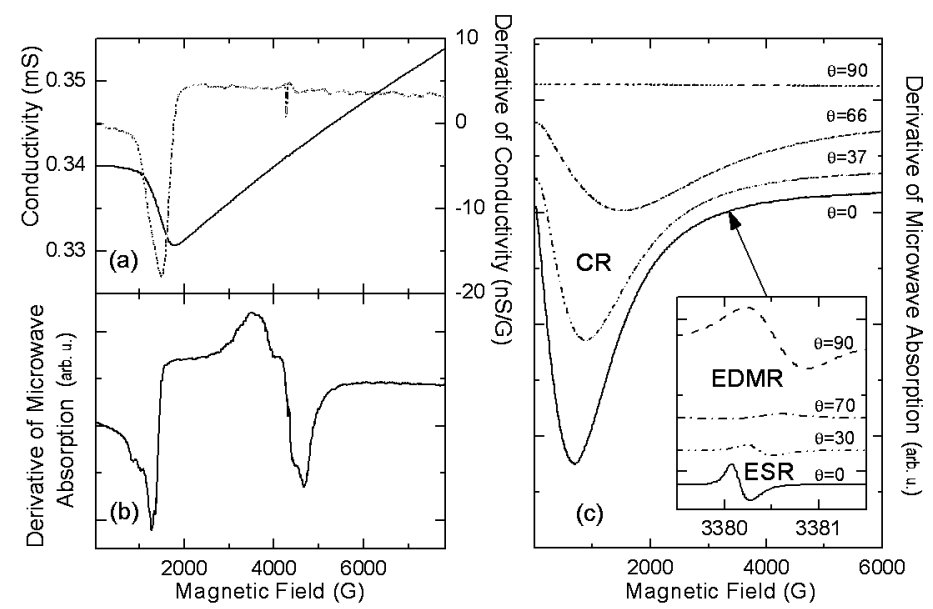

Fig. 1. (a) Dependence of the electrical ac $(71 \mathrm{~Hz})$ conductivity of a (Ga,Mn)As layer on the magnetic field. The dashed line stands for the field derivative of conductivity. (b) Derivative spectrum of the microwave absorption in (Ga,Mn) As. The low field structure corresponds to the magnetoresistance caused by the "flip" of magnetization from the easy in-plane orientation to the external magnetic field direction, applied along the growth axis. A spin wave resonance is seen at higher fields. (c) Derivative of the microwave absorption of $2 \mathrm{D}$ electrons in a $\mathrm{Si} / \mathrm{SiGe}$ quantum well. The broad structure corresponds to the electric absorption at cyclotron resonance (CR). The inset to (c): blow-up of the ESR range. The change of the absorption caused by ESR is by 7 orders of magnitude smaller as compared to the CR absorption.

\subsection{Low frequency conductivity}

Data for the low frequency conductance of a $(\mathrm{Ga}, \mathrm{Mn}) \mathrm{As}$ layer are shown in Fig. 1a as a function of the applied magnetic field. The low frequency conductivity spectra were obtained at $f=71 \mathrm{~Hz}$. The minimum at $H=1400 \mathrm{G}$ corresponds to the "spin flip" - reorientation of the magnetization. As it is known from the analysis of the angular dependence of the magnetic resonance, at low fields the magnetization lies in the film plane. With increasing perpendicular field (parallel to the growth axis) the direction of the magnetization vector "flops" from in-plane orientation and to nearly vertical, at $H=1400 \mathrm{G}$ in this sample. This spin flop 
is well confirmed by the angular dependence of its position: if we tilt the magnetic field with respect to the growth direction then the spin-flip field increases as expected theoretically.

The same structure related to the change of the direction of magnetic moment is also seen in Fig. 1b, where the derivative spectrum of the microwave absorption is shown. The low field structure corresponds to the derivative of the electrical conductivity as shown in Fig. 1a. Therefore we can conclude that this structure reflects the magnetoresistance of the $(\mathrm{Ga}, \mathrm{Mn})$ As layer measured at a frequency $9.44 \mathrm{GHz}$. The high field signal corresponds to a spin wave resonance [6, 7].

In Fig. 1c and d, spectra are shown for 2D electrons in a Si/SiGe QW. In contrast to the $(\mathrm{Ga}, \mathrm{Mn}) \mathrm{As}$ layer, no characteristic structure is observed in the dc or low frequency conductivity on magnetic field [8]. The $9.44 \mathrm{GHz}$ microwave absorption shown in Fig. 1c is dominated, however, by the electrical absorption. The observed structure reflects the derivative of cyclotron resonance absorption. The cyclotron resonance is centered at $600 \mathrm{G}$, which corresponds to an effective mass of $m^{*}=0.19 m_{0}$. The spin resonance is hardly seen at this scale. The inset shows this spectrum with a higher resolution, where a sharp structure, related to the ESR can be well recognized. A similar signal was also seen in dc EDMR measurements [1].

We have not found any microwave induced EDMR signal in (Ga,Mn)As. Neither the difference of conductivity with and without microwave power, nor the field derivative of the ac conductivity under microwave radiation show any signal which could be related to the magnetic resonance. This indicates that the observed resonance shown in Fig. 1b originates from the magnetic dipole absorption only, but not from an EDMR signal which is too small to be measured.

In dc EDMR measurements only the change in conductivity caused by magnetic resonance is monitored while the resonance signal of the microwave absorption is a superposition of the EDMR and magnetic dipole absorption. As it is discussed below the contribution of the direct ESR signal and the indirect contribution of EDMR to the microwave absorption can be distinguished by analyzing the dependence of the signal amplitude on microwave power, $P$. In Fig. 2 the power dependence of the microwave signal amplitude, normalized by $P^{1 / 2}$, is presented. For $2 \mathrm{D}$ electrons in $\mathrm{Si} / \mathrm{SiGe}$ a complex dependence is observed. Only in the very low power range the normalized amplitude is constant. Next it increases and the amplitude is proportional to the $P$ in an intermediate range. Finally, it saturates and decreases at high $P$. The amplitude of the magnetic resonance in $(\mathrm{Ga}, \mathrm{Mn}) \mathrm{As}$ is constant for the whole range of $P$. The weak decrease of the signal at the highest power is caused by sample heating, but not by saturation effects.

\section{Electrically detected magnetic resonance}

In spite of the fact that the magnetic resonance absorption is well pronounced and that the dependence of the sample conductivity on spin structure is well 


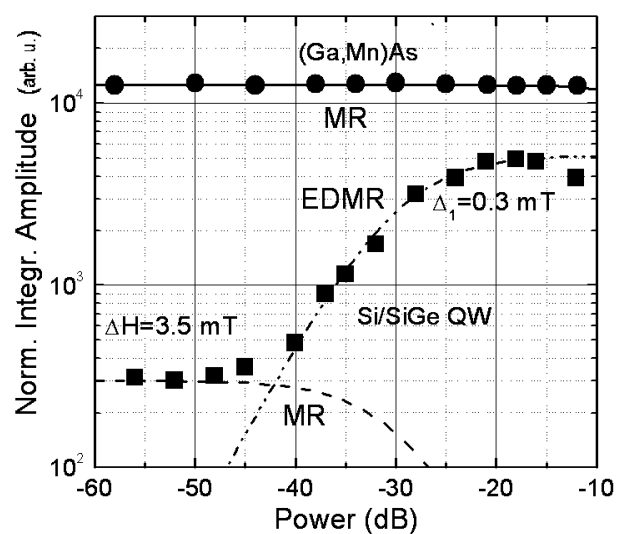

Fig. 2. Integrated amplitude of the resonance signal normalized by $P^{1 / 2}$. The direct magnetic resonance signal has a power independent amplitude in this kind of plot. A linear increase in the signal amplitude is an indication of EDMR. The saturation of signals at a high microwa ve power allows one to evaluate the longitudinal spin relaxation rate, $\Delta_{1}$.

evidenced, no EDMR signal in (Ga,Mn)As was observed. To explain this puzzle we try to model the EDMR signal and verify it by the experimental ESR data on $\mathrm{Si} / \mathrm{SiGe}$. This system was investigated in detail in Ref. [2].

The amplitude of the EDMR signal, $I_{\text {EDMR }}$, is proportional to the dependence of the electrical conductivity, $\sigma$, on spin polarization, $\eta_{\mathrm{s}}$ and on the change of the spin polarization, $\Delta \eta_{\mathrm{s}}(P)$, caused by microwave resonance absorption under microwave power, $P$. For small perturbation of the spin polarization, the EDMR signal amplitude is given by

$$
I_{\mathrm{EDMR}}(P) \propto \sigma(P, H)-\sigma(0, H) \cong \delta_{\mathrm{s}}\left(\eta_{\mathrm{s}}(P, H)-\eta_{\mathrm{s} 0}\right),
$$

where $\eta_{\mathrm{s} 0}=\eta_{\mathrm{s}}(0,0)$ and $\delta_{\mathrm{s}} \equiv \mathrm{d} \sigma / \mathrm{d} \eta_{\mathrm{s}}$.

The derivative $\delta_{\mathrm{s}} \equiv \mathrm{d} \sigma / \mathrm{d} \eta_{\mathrm{s}}$ is a material parameter ruled by the influence of spin-orbit coupling on the electrical conductivity. In both systems investigated this parameter is not negligible. As it is shown in Fig. 1 for $(\mathrm{Ga}, \mathrm{Mn}) \mathrm{As}$ the relative change of the electrical conductivity caused by the change of the direction of magnetization is of the order of a few percent. The ratio $\delta_{\mathrm{s}} / \sigma$, however, is expected to be much bigger since momentum scattering in ferromagnetic (Ga,Mn)As is ruled by spin-flop processes, which lead to negative magnetoresistance [4]. The spin-flop momentum relaxation is ruled by the Fermi surface of minority spins. Therefore, the relative change of electric conductivity can be estimated to be close to $\left|\delta_{\mathrm{s}} / \sigma\right| \cong 1$. The negative magnetoresistance is also seen in Fig. 1, where the resistance decreases in the high field range.

For $2 \mathrm{D}$ electrons in $\mathrm{Si} / \mathrm{SiGe} \mathrm{QW}$ our measurements do not allow to distinguish any noticeable change in magnetoresistance due to a change of spin polarization. For our experimental conditions, where the applied field is of the order 
of a fraction of 1 Tesla, the spin splitting is at least by an order of magnitude smaller than the Fermi energy. Consequently, the equilibrium magnetization is much smaller than its saturation value. In that range of applied fields the Fermi surfaces for both spin orientations are expected to be field independent. The high field magnetoresistance measurements, however, show that the increase in the Pauli magnetization towards its saturation value, where the minority spin subband is not occupied, results in a step-like decrease in the conductivity by a factor of 2 [3]. Thus for the high mobility 2D electron gas in Si QW's in the high magnetic field range we expect $\delta_{\mathrm{s}} / \sigma \cong 1$ but for the low field range, corresponding to our experimental conditions $\delta_{\mathrm{s}} / \sigma \ll 1$.

\subsection{Spin polarization}

The spin polarization under microwave radiation results from the balance of the resonant absorption and spin relaxation. The absorption is proportional to the microwave power and the imaginary part of the magnetic susceptibility, $\chi^{\prime \prime}(H)=\chi_{0} f(P, H)$. For the Lorentzian line shape function

$$
f(p . h)=\frac{1}{\pi(1+p)} \frac{1}{1+\frac{h^{2}}{1+p}},
$$

the steady state spin polarization is

$$
\eta(p . h)=\frac{\eta_{0}}{1+p} \frac{1+h^{2}}{1+\frac{h^{2}}{1+p}} .
$$

Here $h=\left(H-H_{0}\right) / \Delta H$ is a dimension-less magnetic field, centered at the resonance field, $H_{0}$, and it is normalized by the linewidth, $\Delta H$. We define also a dimension-less microwave power, $p=H_{1}^{2} / \Delta H_{1} \Delta H$, where $H_{1}$ is the microwave magnetic field and $\Delta H_{1}$ is the contribution to the linewidth caused by the longitudinal relaxation of the magnetization. The latter is equal to the product of the gyroscopic ratio, $\gamma$, and the longitudinal relaxation rate $\Delta H_{1}=\gamma T_{1}^{-1}$.

Equation (3) shows that the change of the spin polarization at the center of resonance $(h=0)$, proportional to the EDMR signal (see Eq. (1)), is described by

$$
I_{\mathrm{EDMR}} \propto \eta_{\mathrm{s}}(p, H)-\eta_{\mathrm{s} 0}=\frac{p}{1+p} \eta_{0} .
$$

For a small power, where $p \ll 1$, the change of the spin polarization is proportional to $P$ and it saturates when $p \rightarrow 1$, i.e., the square of the microwave field, proportional to the microwave power, approaches the condition $P \propto H_{1}^{2} \rightarrow \Delta H_{1} \Delta H$. In our cavity the microwave field is $H_{1} \cong 1 \mathrm{G}$ at the maximum power of $0.2 \mathrm{~W}$ $(0 \mathrm{~dB})$. The solid line in Fig. 2 is described by Eq. (4). The linear increase and the saturation is well seen. Since the resonance linewidth, $\Delta H$, is known from the direct observation, the analysis of saturation allows one to evaluate the longitudinal spin relaxation. The sharp decay of the signal amplitude at high power is caused by the sample heating by the microwaves. 


\subsection{Signals}

The characteristic dependence on microwave power allows one to distinguish the direct and the electrically detected magnetic resonance signal. The direct signal (normalized, for the technical reasons, by $P^{1 / 2}$ ) is constant in contrast to the EDMR signal which increases with $P$. As seen in Fig. 2, at very low $P$ the EDMR signal of $2 \mathrm{D}$ electrons in $\mathrm{Si} / \mathrm{SiGe} \mathrm{QW}$ becomes too small and only the direct signal is observed.

The constant value of the magnetic resonance of $(\mathrm{GaMn})$ As indicates that the observed signal corresponds to the direct signal, caused by the magnetic dipole absorption, and it has no contribution of an EDMR signal. Moreover, the independence of the signal on $P$ indicates that the observed resonances cannot be saturated at the available microwave power. The lack of the saturation of the (GaMn)As signal allows one to estimate a lower limit of the longitudinal relaxation: $1 \mathrm{G}<\Delta H_{1}$.

On the other hand, since the contribution to the linewidth caused by the longitudinal relaxation cannot exceed the observed linewidth, $\Delta H=\Delta H_{1} / 2+$ $\Delta H_{2} \cong 20 \mathrm{G}$, we can conclude that $1 \mathrm{G}<\Delta H_{1}<40 \mathrm{G}$.

The longitudinal spin relaxation in $(\mathrm{Ga}, \mathrm{Mn}) \mathrm{As}$ is by $2 \div 3$ orders of magnitude shorter as compared to the investigated high mobility $2 \mathrm{D}$ electrons in the $\mathrm{Si}$ quantum well. Moreover, the resonance linewidth is by another $2 \div 3$ orders of magnitude bigger in the case of $(\mathrm{Ga}, \mathrm{Mn})$ As. Together, the product $\Delta H_{1} \Delta H$ is by a factor of about $10^{4}$ bigger, which means that the saturation of the spin polarization occurs at a power which is by five orders of magnitude bigger. It means also that at the same microwave power the change of the spin polarization, $\eta_{\mathrm{s}}\left(P, H_{0}\right)-\eta_{s 0}$, is by a factor $10^{4}$ smaller in the case of $(\mathrm{Ga}, \mathrm{Mn}) \mathrm{As}$. Therefore, we relate the lack of the EDMR signal in $(\mathrm{Ga}, \mathrm{Mn})$ As to the fast spin relaxation.

\section{Conclusions}

A comparison of the results of two very different materials leads us to the following conclusions:

(i) the change of the electrical conductivity caused by resonance microwave absorption (EDMR) is ruled by spin dependent electron scattering, proportional to a change of spin polarization;

(ii) a pronounced EDMR signal can be expected when the spin polarization can be effectively modified, i.e., when spin relaxation is slow as compared to resonance absorption;

(iii) in contrast to classical magnetic resonance, the EDMR signal is independent of the sample volume. Consequently, it can be also investigated in nano-structures. 


\section{Acknowledgments}

This work was supported by the State Committee for Scientific Research Grant No. PBZ 044/P03/2001 and 2 P03B 05423 in Poland and by the FWF and OeAD in Austria.

\section{References}

[1] C.F. Graeff, M.S. Brandt, M. Stutzmann, M. Holzmann, G. Abstreiter, F. Schäffler, Phys. Rev. B 59, 13242 (1999).

[2] Z. Wilamowski, W. Jantsch, Phys. Rev. B 69, 035328 (2004).

[3] V.M. Pudalov, M.E. Gershenson, H. Kojima, G. Brunthaler, A. Prinz, G. Bauer, Phys. Rev. Lett. 91, 126403 (2003).

[4] H. Ohno, J. Magn. Magn, Mater. 200, 110 (1999).

[5] T. Dietl, H. Ohno, F. Matsukura, J. Cibert, D. Ferrand, Science 287, 1019 (2000).

[6] O.M. Fedorych, Z. Wilamowski, M. Potemski, M. Byszewski, J. Sadowski, Acta, Phys. Pol. A 103, 607 (2003).

[7] T.G. Rappoport, P. Redlinski, X. Liu, G. Zarand, J.K. Furdyna, B. Janko, Phys. Rev. B 69, 125213 (2004).

[8] F. Schäffler, Semicond. Sci. Technol. 12, 1515 (1997). 